\title{
SÍNDROME DE DESMORALIZACIÓN COMO ALTERNATIVA DIAGNÓS- TICA AL ESTADO DE ÁNIMO DEPRIMIDO EN PACIENTES PALIATIVOS
}

\author{
DEMORALIZATION SYNDROME AS AN ALTERNATIVE DIAGNOSTIC TO DEPRESSED \\ MOOD IN PALLIATIVE PATIENTS
}

David Rudilla', Pilar Barreto', Amparo Oliver ${ }^{3}$ y Laura Galiana ${ }^{3}$

' Unidad de Hospitalización a Domicilio, Servicios de Hospitalización Domiciliaria, Consorcio Hospital General Universitario, Valencia, España

2 Departamento de Personalidad, Evaluación y Tratamientos Psicológicos, Universidad de Valencia, Valencia, España

${ }^{3}$ Departamento de Metodología de las Ciencias del Comportamiento, Universidad de Valencia, Valencia, España

Resumen

El estado de ánimo de los pacientes paliativos es uno de los objetivos fundamentales en la atención profesional a los mismos. Distintos autores han estudiado dicho estado de ánimo tomando como base definiciones de la depresión tal como se entiende en la población general. Son muchas las voces y los datos de investigación que apuntan a la necesidad de adecuar la investigación a la situación del paciente en final de vida. Quizá una de las ideas más interesantes es la planteada por Frank y desarrollada por Kissanne y colaboradores, proponiendo la diferenciación entre depresión y desmoralización. Siguiendo esta línea de pensamiento, la desmoralización reflejaría mejor la situación observada en estos pacientes, permitiendo un mejor diagnóstico diferencial y promoviendo un afinamiento mayor en las intervenciones. El síndrome de desmoralización vendría definido como la presencia de desánimo, desaliento, desamparo, pérdida de sentido de la vida y fracaso, sin que los síntomas somáticos propios de la conceptualización de la depresión tengan un peso importante. Teniendo en cuenta esta definición, las intervenciones psicológicas tendrían unos objetivos operativos distintos al tratamiento de depresión, obteniéndose resultados en el bienestar del paciente paliativo. En este trabajo se realiza un planteamiento crítico
Abstract

The emotional state of palliative patients is one of the main aims in their professional attention. Several authors have approach this matter using depression definitions as defined for the general population. There are also many expert professional opinions and research data pointing to the need for adequate research at the patient's end of life situation. Perhaps one of the most interesting ideas is that one initially expressed by Frank and developed by Kissanne et al., suggesting differentiation between depression and demoralization. Following this line of thought, demoralization would more accurately reflect the situation observed in these patients, allows a better differential diagnosis and promotes more tailored interventions. Demoralization syndrome would be defined as the presence of discouragement, despair, helplessness, loss of meaning in life and feelings of failure. That approach leaves aside somatic symptoms of depression scores. This work exposes a critical view and being thought-provoking about current interventions in palliative care.

Keywords: Depression, palliative care, treatment, demoralization.

Correspondencia:

David Rudilla

Consorcio Hospital General Universitario Valencia. Avenida Tres Cruces, 2 - 46014 - Valencia

E-mail: rudilla_dav@gva.es 
y se invita a reflexionar sobre las intervenciones psicológicas que actualmente se llevan a cabo en pacientes paliativos.

Palabras clave: Depresión, cuidados paliativos, tratamiento, desmoralización.

\section{PLANTEAMIENTO DEL PROBLEMA}

La depresión en pacientes paliativos ha sido descrita hace ya mucho tiempo en el marco específico del modelo de adaptación en etapas planteado por Kübler-Ross ${ }^{(1)}$. Como es sabido, desde este enfoque la depresión es la cuarta etapa que vive el paciente paliativo, en la que comienza a ser consciente de la seguridad de la muerte. En esta situación, la persona puede permanecer en silencio, rechazar visitas y pasar mucho tiempo llorando y lamentándose. En ocasiones, este proceso puede promover incluso que el paciente se aleje de todo sentimiento de amor y cariño. Cuando la autora se plantea el abordaje de esta situación, la herramienta que enfatiza es la normalización de dicha etapa, con indicación explícita de evitar "mensajes de ánimo discordantes afectivamente" ya que el paciente necesita transcurrir por esa etapa como parte de su proceso de adaptación.

Es frecuente que los profesionales sanitarios que trabajan en cuidados paliativos etiqueten el estado de ánimo descrito como "depresión". Teniendo en cuenta el síndrome de depresión tal como es definido en el DSM-V (ver tabla 1) este diagnóstico en la situación descrita anteriormente no parece el más adecuado, ya que habitualmente se detecta una sintomatología que no encaja totalmente con la definición planteada en el manual. La elevada presencia de sintomatología somática, también asociada al proceso de enfermedad, hace difícil un diagnóstico diferencial adecuado. Además, algunos de los síntomas relaciona- dos con este diagnóstico como la pérdida de sentido de la vida o la sensación de fracaso, estarían mejor enmarcados en el contexto de las necesidades espirituales, cuyo abordaje se considera fundamental en el tratamiento sintomático-paliativo, sin consideración de los mismos como síntomas de psicopatología.

La probable presencia de este síndrome en pacientes paliativos es habitualmente objetiva para los profesionales sanitarios. Así, en un estudio llevado a cabo en una unidad de cuidados paliativos ${ }^{(2)}$ se encontró que un número elevado de solicitudes de intervención psicológica hacían referencia a circunstancias que se calificaron como "emocionales extremas", refiriendo la tristeza extrema como uno de los componentes clave. Si entendemos que el diagnóstico es poco ajustado, la confusión respecto al impacto de la tristeza asociada al síndrome depresivo afecta tanto la calidad de vida de los pacientes y las interacciones con la familia y amigos, como a la efectividad del tratamiento global (síntomas físicos como dolor, disnea, etc.). Se entiende pues que si bien el diagnóstico inicial para cualquier profesional podría ser de un cuadro depresivo, existe confusión en la literatura actual sobre la pertinencia del diagnóstico de las alteraciones de tipo afectivo en pacientes en la fase final de la vida.

Por otro lado, contamos con datos de investigaciones en los que se muestra que tras las intervenciones psicológicas los resultados obtenidos, medidos con cuestionarios de amplio consenso como el HADS, las puntuaciones en "depresión" no dis- 


\section{Tabla 1. Criterios diagnósticos para un episodio depresivo mayor. DMS-V(80)}

A. Presencia de cinco (o más) de los siguientes síntomas durante un período de 2 semanas, que representan un cambio respecto a la actividad previa; uno de los síntomas debe ser 1: estado de ánimo depresivo o 2: pérdida de interés o de la capacidad para el placer.

Nota: No se incluyen los síntomas que son claramente debidos a enfermedad médica o las ideas delirantes o alucinaciones no congruentes con el estado de ánimo.

1. Estado de ánimo depresivo la mayor parte del día, casi cada día según lo indica el propio sujeto (p. ej., se siente triste o vacío) o la observación realizada por otros (p. ej., Ilanto). En los niños y adolescentes el estado de ánimo puede ser irritable

2. Disminución acusada del interés o de la capacidad para el placer en todas o casi todas las actividades, la mayor parte del día, casi cada día (según refiere el propio sujeto u observan los demás)

3. Pérdida importante de peso sin hacer régimen o aumento de peso (p. ej., un cambio de más del $5 \%$ del peso corporal en 1 mes), o pérdida o aumento del apetito casi cada día. Nota: En niños hay que valorar el fracaso en lograr los aumentos de peso esperables

4. Insomnio o hipersomnia casi cada día

5. Agitación o enlentecimiento psicomotores casi cada día (observable por los demás, no meras sensaciones de inquietud o de estar enlentecido)

6. Fatiga o pérdida de energía casi cada día

7. Sentimientos de inutilidad o de culpa excesivos o inapropiados (que pueden ser delirantes) casi cada día (no los simples autorreproches o culpabilidad por el hecho de estar enfermo)

8. Disminución de la capacidad para pensar o concentrarse, o indecisión, casi cada día (ya sea una atribución subjetiva o una observación ajena)

9. Pensamientos recurrentes de muerte (no sólo temor a la muerte), ideación suicida recurrente sin un plan específico o una tentativa de suicidio o un plan específico para suicidarse

B. Los síntomas no cumplen los criterios para un episodio mixto.

C. Los síntomas provocan malestar clínicamente significativo o deterioro social, laboral o de otras áreas importantes de la actividad del individuo.

D. Los síntomas no son debidos a los efectos fisiológicos directos de una sustancia (p. ej., una droga, un medicamento) o una enfermedad médica (p. ej., hipotiroidismo).

E. Los síntomas no se explican mejor por la presencia de un duelo (p. ej., después de la pérdida de un ser querido), los síntomas persisten durante más de 2 meses s o se caracterizan por una acusada incapacidad funcional, preocupaciones mórbidas de inutilidad, ideación suicida, síntomas psicóticos o enlentecimiento psicomotor.

minuyen, sino que aumenta ${ }^{(3-8)}$. Al mismo tiempo, los resultados de varios trabajos encuentran mayores niveles de significado en la vida, calidad de vida y el bienestar espiritual tras la intervención psicológica, aunque no se determinan los mismos be- neficios sobre la depresión. En esta línea, Tremblay y Breitbart ${ }^{(9)}$ afirman que los síndromes psiquiátricos, como depresión, ansiedad, confusión, ideación suicida, y el deseo de una muerte apresurada o la solicitud de suicidio asistido se producen 
en un porcentaje significativo en pacientes paliativos en su fase final. Sin embargo, Lichthenthal y colaboradores ${ }^{(10)}$ no encontraron que las tasas de trastornos depresivos y de ansiedad aumentasen a medida que los pacientes se acercaban a la muerte, señalando que el desafío al que se enfrentan los clínicos se centra en distinguir la angustia adaptativa o normativa de la psicopatología. Dadas las implicaciones de cara al tratamiento tan significativas, resulta imprescindible detectar adecuadamente la tristeza y la ansiedad esperadas y debidas al proceso de adaptación o al deterioro clínico diferenciándolas de las psicopatologías reales.

A partir de esta información cabe preguntarse ${ }_{i}$ Es posible que mejore la calidad de vida de un paciente y a la vez percibir empeoramiento en el estado de ánimo? ¿Es el concepto de "depresión", tal como se utiliza habitualmente en otros ámbitos, aplicable al paciente paliativo si en muchos casos, tal como se desprende de planteamientos como el de Kübler-Ross, hablamos de una respuesta normalizada a la situación de terminalidad? ¿Existen evidencias que señalen la existencia de un cuadro sintomático propio como es el síndrome de desmoralización?

\section{Objetivos de la revisión}

El objetivo de este trabajo es doble. Por una parte, llevar a cabo una revisión de la investigación sobre la prevalencia de la depresión en el paciente paliativo, atendiendo tanto a los planteamientos teóricos desarrollados en los últimos años como a resultados obtenidos. Además, en este objetivo se tendrán en cuenta los resultados de los tratamientos farmacológicos y psicoterapias empleados. En síntesis, se pretende profundizar en el conocimiento del cuadro sintomático depresivo en el paciente paliativo.

El segundo objetivo se dirige a revisar las evidencias teóricas y empíricas que apoyen la existencia del Síndrome de Desmoralización en pacientes paliativos como categoría diagnóstica más adecuada. Tal evidencia facilitaría ajustar objetivos terapéuticos concretos y por lo tanto, los tratamientos pertinentes alineados a la consecución de esos objetivos.

\section{DESARROLLO DEL TEMA}

El diagnóstico de depresión en cuidados paliativos

Ante la presencia de síntomas depresivos es importante en la valoración clínica, determinar en primer lugar si tales síntomas son adaptativos o no. La dificultad de diagnosticar el trastorno depresivo en pacientes con cáncer u otras enfermedades graves ha sido documentada en diferentes trabajos ${ }^{(11-16)}$, una gran mayoría de ellos coincide en considerar la influencia en este proceso diagnóstico de factores relativos a las siguientes cuestiones: (1) personal sanitario (p.ej., empleo de indicadores poco fiables de la depresión, sobrevaloración de la sintomatología somática, falta de valoración por escasez de conocimientos, de la vida afectiva de los pacientes, etc.); (2) a las características de los pacientes (p.ej., expresión de bloqueo, dificultad para informar de sus síntomas depresivos, resistencia a buscar tratamiento por los síntomas psicológiCos); (3) las características de la propia enfermedad (p.ej., dificultad para distinguir entre los síntomas somáticos que son consecuencia de la enfermedad y los que lo son de la depresión); (4) características de la depresión (p.ej., dificultad para distinguir entre depresión clínica y disforia subclínica o respuesta «normal»a una situación estresante); (5) condiciones sanitarias (p.ej., elevado número de pacientes por profesional, carencia de estructuras adecuadas para deliberar sobre los problemas personales y emocionales). 
Respecto a la frecuencia del problema que nos ocupa, diversos autores ponen de manifiesto la presencia de trastornos de tipo depresivo en pacientes paliativos ${ }^{(15,16)}$. Alrededor del 25\% de los pacientes con cáncer experimentan síntomas depresivos, con una prevalencia que aumenta hasta el $77 \%$ en aquellos con enfermedad avanzada $^{(17)}$. Así, Mitchell et al. ${ }^{(15)}$ identificaron 24 estudios sobre trastornos de estado de ánimo en unidades o servicios de cuidados paliativos de 7 países, de ellos se deduce que la depresión tuvo una prevalencia de entre el 5,1\% y el 30,1\%. El análisis meta-analítico cifró esta prevalencia en un $16,5 \%$ (con un intervalo de confianza del 95\%:13,1-20,3). Estudios más recientes han arrojado mayores tasas de prevalencia, como por ejemplo en el caso de Rajmohan y Kumar ${ }^{(16)}$ quienes encontraron una tasa de prevalencia del $36 \%$. Por tanto queda fuera de toda duda la importancia de prestar atención a este fenómeno.

Los síntomas depresivos que se estudian con profundidad en cuidados paliativos se refieren al bajo estado de ánimo, la desesperanza, la ansiedad, la focalización continua en la situación actual, el aislamiento social, la inquietud, la interrupción del sueño, los cambios en el apetito y el peso, los sentimientos de inutilidad, los sentimientos de culpa y los pensamientos de muerte como solución ${ }^{(17)}$. Es importante, además, que este conjunto de manifestaciones se entienda desde la perspectiva de la situación global que afecta al paciente. Resulta interesante también la información aportada por Wilson et al. ${ }^{(18),}$ quienes identificaron los factores de riesgo de depresión en pacientes paliativos (ver tabla 2).

\section{Tabla 2. Factores de riesgo de depresión en pacientes paliativos(18)}

1) Diagnóstico de enfermedad terminal

2) Determinados tipos de cáncer: páncreas, tumores cerebrales

3) Comorbilidades: hipotiroidismo, enfermedad arterial coronaria, degeneración macular, diabetes mellitus, enfermedad de Alzheimer, Enfermedad de Parkinson, esclerosis múltiple, Huntington.

4) Discapacidad física

5) Control de síntomas inadecuado

6) Anomalías metabólicas: hipercalcemia, toxinas generadas por el tumor, uremia, medicación con vida media anormal

7) Medicaciones: anfotericina, hipertensivos, H2-bloqueantes, metoclopramida, fármacos citotóxicos, corticoides, interferon, interleukin.

8) Radioterapia

9) Malnutrición

10) Pérdida cognitiva

11) Historia previa de depresión

12) Historia familiar de depresión

13) Edad del paciente, mayor riesgo en pacientes jóvenes

14) Petición de mantener o retirar tratamiento

15) Petición de suicidio asistido

16) Consumo de sustancias

17) Escaso apoyo social

18) Falta de relaciones interpersonales cercanas

19) Problemas económicos 
Basándose en el conjunto de factores de riesgo, se han desarrollado diversos instrumentos de medida para la evaluación del paciente, que permitirán llevar a cabo la intervención más adecuada.

Cuestionarios de evaluación de la depresión en pacientes paliativos

En los distintos estudios revisados, los cuestionarios de evaluación de la depresión en pacientes paliativos más frecuentemente utilizados son:

- Escala de depresión postnatal de Edimburgo, EPDS ${ }^{(19)}$. Escala de 10 ítems, con un punto de corte de 13 puntos para identificar depresión. Incluye ítems relacionados con la tristeza subjetiva, la culpa, la desesperanza, y los sentimientos autopunitivos, siendo éstos independientes de la discapacidad física. Validada en una muestra de 100 pacientes con cáncer avanzado(20).

- Escala de Ansiedad y Depresión Hospitalaria, $\operatorname{HADS}^{(21,22)}$. Desarrollada para diagnosticar ansiedad y depresión en población médica general, es la más empleada en cuidados paliativos, supera el principal problema de la evaluación de la depresión en la enfermedad avanzada: la influencia de los síntomas causados por la enfermedad física en los resultados de las escalas de depresión y de ansiedad ${ }^{(23)}$. Es importante señalar la inconsistencia de resultados en los trabajos que han estudiado su idoneidad como prueba de screening en pacientes con cáncer avanzado ${ }^{(25,26,27)}$; dichos estudios tienen a sobreestimar la prevalencia ya que se han reducido especificidad y alta sensibilidad ${ }^{(24)}$.

- Entrevista de un ítem, y entrevista de dos ítems ${ }^{(12,28,29)}$. En la primera se pregunta al sujeto si está deprimido, y en la segunda se añade una pregunta sobre la pérdida de intereses. Estas dos preguntas cubren el núcleo de los crite- rios de la depresión del DSM-V y sirven como una primera aproximación a la posible existencia de depresión en los enfermos graves.

- Inventario de Depresión de Beck (BDI, $B D I-I I)$. Es un autoinforme de lápiz y papel compuesto por 21 ítems con respuesta tipo Likert. El inventario inicialmente propuesto por Beck y sus versiones posteriores han sido los instrumentos más utilizados para detectar y evaluar la gravedad de la depresión. De hecho, es el quinto test más utilizado por los psicólogos españoles ${ }^{(30)}$. Sus ítems no se derivan de ninguna teoría concreta acerca del constructo medido, sino que describen los síntomas clínicos más frecuentes de los pacientes psiquiátricos con depresión.

- Varios investigadores han sugerido que preguntar a los pacientes si se encuentran deprimidos era un indicador útil para diagnosticar la depresión ${ }^{(12,31)}$. No obstante las limitaciones a esta metodología están relacionadas con la escasa discriminación entre distintos síntomas de los que configuran el diagnóstico de depresión.

- Las Escalas de Valoración Analógica (EVA) se han empleado con pacientes con cáncer ${ }^{(32,33)}$. Como limitación se ha descrito que la experiencia subjetiva del paciente puede tanto infrapuntuar como sobrepuntuar su respuesta, además las diferencias en las apreciaciones perceptivas pueden contribuir a tales desajustes.

Respecto a la evaluación, Hotopf et al. (24) Ilevaron a cabo un estudio en el que tras identificar cuatro aproximaciones empleadas para detectar depresión en pacientes al final de la vida, concluyeron que los niveles de prevalencia de la depresión empleando criterios de sustitución no diferían de las obtenidas a partir de descripciones convencionales de la misma. 
El empleo de instrumentos de evaluación de la depresión en pacientes paliativos parece ofrecer en muchos casos grandes dificultades y arroja resultados difícilmente asumibles desde la visión clínica, produciendo la sensación de que estos instrumentos parecen medir algo distinto a lo que realmente se está observando. Juliao y Barbosa ${ }^{(23)}$ señalan que siendo la depresión la patología psíquica más común en cuidados paliativos, la literatura científica existente presente limitaciones francas, especialmente en relación con las indicaciones para el tratamiento y criterios de diagnóstico utilizados. Chochinov et al. ${ }^{(34)}$ mostraron uno de los principales problemas en la evaluación de las intervenciones, a saber, el uso de instrumentos sensibles a los cambios que se buscan, dada la especificidad tanto del cuadro sintomático como de la situación de los pacientes. Del mismo modo que sucede con la ansiedad, en los últimos años se está trabajando en otros instrumentos que puedan ser sensibles a las respuestas de este tipo de pacientes, como el BSI-18 $8^{(35,36)}$ o el termómetro sobre el sufrimiento ${ }^{(22,37,38)}$. Todo parece indicar que los signos y síntomas como anhedonia, pérdida / ganancia de peso, anorexia, insomnio y retraso psicomotor pueden ser consecuencia de una enfermedad médica avanzada y no depresión. Otros indicadores clínicos tienen un mayor valor predictivo para la depresión en esta población: sentimientos de desesperanza, impotencia, inutilidad, culpabilidad y pensamientos suicidas ${ }^{(23,38)}$.

En esta línea, el síndrome de desmoralización planteado por Kissane, Clarke y Street $^{(38)}$ parece englobar las características anímicas específicas de los pacientes en la fase final de la vida. La principal diferencia entre la depresión y el síndrome de desmoralización se encuentra en la consideración que en tales diagnósticos tienen los síntomas somáticos. Estos son muy importantes para el diagnóstico de la primera con el mismo peso que aportan los cognitivos. En el planteamiento de la desmoralización en pacientes paliativos se hace mayor hincapié en los aspectos emocionales y experienciales dado que los síntomas físicos pueden indicar naturaleza patológica, es decir, con una base orgánica como causante, en lugar de una cognitiva. Parece pues que el síndrome de desmoralización supondría un mejor reflejo del estado de ánimo del paciente paliativo, donde la desesperanza, o los sentimientos de dependencia, aspectos más vivenciales, de carácter cognitivo o emocional, se perfilan como determinantes más decisivos que la pérdida de peso o el insomnio, tanto en la descripción de su estado de ánimo como en el diagnóstico. Por otra parte, la anhedonia supondría una característica diferencial entre ambos cuadros clínicos, ya que la capacidad de disfrutar continua presente en una gran proporción de pacientes desmoralizados y no en los deprimidos. Esto explicaría el beneficio que parecen aportar los psicoestimulantes en pacientes paliativos $^{(39)}$.

La importancia de distinguir entre estos cuadros sintomáticos es incuestionable, pues determinará el tratamiento tanto a nivel farmacológico como psicoterapéutico.

\section{Desmoralización}

Tal y como se ha comentado, existe evidencia empírica que apoya la existencia de un cuadro sintomático de tipo depresivo diferente al que se exhibe en otras alteraciones del estado de ánimo ${ }^{(3,4,14,40-46)}$ caracterizado por un estado psíquico cuyos pilares básicos son la desesperanza (pérdida de esperanza generalizada, y específica), la impotencia, la pérdida de significado, el malestar existencial, el pesimismo, cierto grado de soledad y deterioro en la capacidad de afrontamiento que ocurre en pacientes con enfermedad física o mental, concretamente en aquellos que 
sienten amenazada su vida o integridad física $^{(47)}$. La presencia de estos síntomas se ha considerado "normal" o "adaptativa" en pacientes paliativos, Ilegándose a proponer por parte de los autores, su inclusión en el Eje IV del DSM V, como un factor psicosocial.

Estos resultados e ideas conducen a la necesidad de ahondar más en la posibilidad de delimitar y estudiar en profundidad el denominado síndrome de desmoralización en el paciente paliativo. La diferencia básica que algunos autores encuentran con la acepción clásica de depresión se refiere a que, pese a los síntomas afectivos negativos, los sujetos con síndrome de desmoralización mantienen la capacidad de interactuar con el ambiente y de disfrutar el presente, aunque sea sin entusiasmo, dato que estaría relacionado con la reducción de los niveles de ansiedad que sí son esperables tras las intervenciones psicoterapéuticas dirigidas a reducirlos ${ }^{(48)}$.

Notas históricas sobre el síndrome de desmoralización

Desde el Antiguo Testamento y los escritos de los griegos clásicos hasta la ac- tualidad, diversos autores han reconocido de algún modo la presencia del síndrome de desmoralización en las personas bajo determinadas circunstancias. De acuerdo con Clarke, Smith, Dowe, McKenzie y Hermna $^{(48)}$ el concepto de desmoralización actual fue originalmente propuesto por Jerome Frank quien lo describió como impotencia, aislamiento, desesperación, enajenación, rechazo y baja autoestima ${ }^{(49)}$. Esta aproximación la llevó a cabo refiriéndose al contexto de la psicoterapia de pacientes psiquiátricos. El desarrollo posterior del estudio sobre el síndrome de desmoralización se ha ido refiriendo a su presencia en pacientes con enfermedades orgánicas. En la tabla 3 pueden contemplarse distintos acercamientos a este síndrome.

Aunque muchos de los descriptores de la Tabla 3 pueden Ilevar a la consideración de diagnóstico de la depresión, de acuerdo con dichas aproximaciones o definiciones, hay claras diferencias entre los trastornos etiquetados como desmoralización y los del estado de ánimo. Por ejemplo, de Figueiredo ${ }^{(50)}$ hace hincapié en que la desmoralización se caracteriza por sentimientos de incompetencia subjetiva e impotencia, mientras que la depresión se caracteriza por anhedonia

Tabla 3. Aproximaciones a síntomas de desmoralización

\begin{tabular}{|c|c|c|}
\hline Autor & $\begin{array}{l}\text { Año de } \\
\text { publicación }\end{array}$ & Descripción \\
\hline $\mathrm{D}^{\prime} \operatorname{Arcy}^{(81)}$ & 1982 & $\begin{array}{l}\text { Ansiedad, tristeza, desesperanza, impotencia, falta de } \\
\text { autoestima }\end{array}$ \\
\hline de Figueiredo ${ }^{(49)}$ & 1993 & Distrés, incompetencia subjetiva \\
\hline Kissane et al. ${ }^{(38)}$ & 2001 & Desesperanza, pérdida del sentido, distrés existencial \\
\hline Clarke et al. ${ }^{(82)}$ & 2003 & $\begin{array}{l}\text { Ansiedad, aprehensión, incapacidad de afrontamiento, pérdida } \\
\text { de confianza, impotencia, desesperanza }\end{array}$ \\
\hline Griffith et al. ${ }^{(83)}$ & 2005 & Desesperación, impotencia, sensación de aislamiento \\
\hline Clarke et al. ${ }^{(84)}$ & 2006 & $\begin{array}{l}\text { Incapacidad de afrontamiento, impotencia, desesperanza, } \\
\text { autoestima disminuida. }\end{array}$ \\
\hline
\end{tabular}


o falta de placer en experiencias. Además, Clarke y Kissane ${ }^{(47)}$ explican que la capacidad hedónica se conserva en el desmoralizado, pero no en el deprimido. También se pueden observar otras diferencias como las señaladas a partir la investigación empírica, por Clarke, Smith, Dowe, McKenzie y Hermna ${ }^{(48)}$. Estos autores encontraron que la desmoralización se asoció con un afrontamiento de evitación, mientras que en la depresión se daba fundamentalmente anhedonia (expresada en retraimiento, es decir, menor número de actividades sociales y de relaciones cercanas). Estas impresiones y datos ponen de relieve que la desmoralización, aunque esté asociada a disforia, no es equivalente a cualquier forma de depresión reflejada en el DSM-V.

Así pues, la desmoralización puede ser entendida como una condición que resulta de un conflicto existencial. Se manifiesta con síntomas como la desesperanza y la impotencia, causados por la pérdida de propósito y sentido de la vida. Esta expresión de significado de la vida de la persona puede llevar incluso al deseo anticipado de la muerte.

\section{Evaluación de la desmoralización}

Para la evaluación de la desmoralización se han desarrollado una serie de instrumentos:

- Entrevista Epidemiología Psiquiátrica de Investigación, PERI(51). Consta de 26 ítems que representan sentimientos de desmoralización, y son consistentes con la definición de desmoralización de Frank ${ }^{(48)}$ correspondiéndose con las 8 subescalas del PERI-D.

- La Escala de Desmoralización, PERI$D^{(52)}$. Consta de 102 ítems que representan acontecimientos relacionados con 8 subescalas: baja autoestima, desesperanza, pensamiento confuso, pavor (miedo a perder el control y volverse loco), ansiedad, tristeza, síntomas psicofísicos, y percepciones de salud física pobre.

- Escala de Desmoralización, ED ${ }^{(53)}$. Es un cuestionario de autoinforme con 24 ítems tipo Likert (rango de puntuación de 0-96) relacionados con 5 factores: pérdida del sentido de la vida, desánimo, desaliento, desamparo y sensación de fracaso.

- Escala basada en los "Criterios diagnósticos de Investigación Psicosomática", $D C P R^{(54)}$. Dirigida al diagnóstico de alteraciones psicosomáticas, engloba y atiende la desmoralización. Se trata de una entrevista estructurada para el diagnóstico de síndromes psicosomáticos, entre los que se encuentra la desmoralización, cuenta con 5 ítems de los 58 que componen la escala. Mantiene el planteamiento de que los procesos cognitivos son una causa directa de enfermedad física

- Inventario de Incompetencia Subjetiva, $I I S^{(55)}$. Cuestionario de creación reciente de 12 ítems (rango de puntuación 0-36) articulado a través de dos factores: incompetencia básica e incompetencia severa.

Tres de los instrumentos cuentan con propiedades psicométricas adecuadas, aplicables en pacientes paliativos. Así para la ED se han encontrado propiedades psicométricas aceptables a través de 5 estudios $^{(56)}$. No obstante, Constantini et $a^{(56)}$ señalan que la estructura de los factores obtenida a partir de los estudios con la escala no es conclusiva por lo que se requiere un mayor número de análisis. En cuatro de los cinco estudios de validación se encontró una validez discriminante con depresión que iba del $6 \%$ al $23 \%$. Las propiedades del DCPR informadas se centran en la validez discriminante con respecto al constructo depresión. En síntesis informan de que el $71 \%$ de los pacientes clasifica- 
dos como desmoralizados no encajaban en el diagnóstico de depresión. En cuanto al IIS sólo hemos podido encontrar un estudio que informe de sus propiedades psicométricas, por lo que la validación se considera preliminar. En este estudio la fiabilidad fue de .90. Constantini et al(56) encontraron que los datos de aquellos estudios en los que la desmoralización se había medido con el DCPR, muestran que la presencia de este trastorno era significativamente más alta que la encontrada por la ED. La causa posiblemente se encuentre en que los criterios de desmoralización en ambos instrumentos son diferentes, por lo que evalúan un cuadro diferente. El análisis de la validez discriminante en los estudios de validación de la Escala de Desmoralización, encuentra tanto relaciones como diferencias entre los pacientes con diagnóstico de desmoralización y depresión clínica (ver tabla 4).
Especialmente importantes son los resultados en el grupo de pacientes que pueden presentar síndrome de desmoralización pero no pueden ser diagnosticados de depresión. De hecho, la falta de presencia de anhedonia sería el síntoma que descartaría inicalmente el diagnóstico de depresión aunque aparezcan síntomas de tristeza, desesperanza e incluso deseo de morir.

\section{Características del Síndrome de Desmorali- zación en pacientes paliativos}

Del conjunto de trabajos que se han desarrollado para el estudio de este síndrome, Kissane et al. ${ }^{(57)}$ identificaron diversos factores de riesgo, de protección y de predisposición (ver tabla 5) a la desmoralización.

La desmoralización, igual que ocurre con la depresión, puede llegar a pasar desapercibida en los enfermos en fase avanza-

\section{Tabla 4. Resultados de validez discriminante en la validación de la Escala de Desmoralización}

\begin{tabular}{|c|c|c|c|}
\hline Estudio & $\begin{array}{c}\text { País / } \\
\text { Traducción }\end{array}$ & Validez convergente & Validez discriminante \\
\hline Constantini et al. ${ }^{(56)}$ & Italia & BDI, MAC & $\begin{array}{l}6 \%-20 \% \text { Alta desmoralización } \\
\text { pero no depresión clínica }\end{array}$ \\
\hline Kissane et al. ${ }^{(47)}$ & Australia & $\begin{array}{c}\text { McGill QOL, PHQ, } \\
\text { BDI, BHS, HOPES, } \\
\text { SAHD }\end{array}$ & $\begin{array}{l}7 \%-14 \% \text { Alta desmoralización } \\
\text { pero no depresión clínica }\end{array}$ \\
\hline Hung et al ${ }^{(85)}$ & Taiwan & BHS, McGill QOL & $\begin{array}{l}23,4 \% \text { Alta desmoralización pero } \\
\text { no depresión clínica }\end{array}$ \\
\hline Mehnert et al. ${ }^{(41)}$ & Alemania & $\begin{array}{l}\text { DT, PHQ, BHS, } \\
\text { SAHD, McGill } \\
\text { QOL, HOPES }\end{array}$ & $\begin{array}{l}5 \%-20 \% \text { Alta desmoralización } \\
\text { pero no depresión clínica }\end{array}$ \\
\hline Mullane et al. ${ }^{(86)}$ & Irlanda & $\begin{array}{c}\text { BDI, PHQ, BHS, } \\
\text { SAHD, McGill } \\
\text { QOL, HOPES }\end{array}$ & $\begin{array}{l}2,1 \%-5,2 \% \text { Alta desmoralización } \\
\text { pero no depresión clínica }\end{array}$ \\
\hline
\end{tabular}

Notas: $\mathrm{BDI}=$ Inventario de Depresión de Beck; MAC= Minimental Ajuste al Cáncer; McGill QOL= McGill Qualitity of life Scale; PHQ= Cuestionario de Salud del Paciente de 9 ítems; BHS= Escala de Desesperanza de Beck; HOPES = Hunter Opinion and Personal Expectations Scale; SAHD = Schedule of Attitudes Toward Hastened Death; DT= Termómetro de Distréss; LAP-R= Life Attitude Profile-Revised. 


\section{Tabla 5. Factores relacionados con el Síndrome de Desmoralización en pacientes paliativos ${ }^{(57)}$}

\begin{tabular}{|c|c|c|}
\hline Factores de riesgo & Factores de protección & Factores de predisposición \\
\hline $\begin{array}{l}\text { - Jóvenes } \\
\text { - Alteración corporal / } \\
\text { desfiguración } \\
\text { - Incapacidad física } \\
\text { - Incapacidad mental } \\
\text { - Dependencia de otros y } \\
\text { sensación de ser una carga } \\
\text { - Ideas suicidas } \\
\text { - Aislamiento social } \\
\text { - Amenaza de perder la dignidad } \\
\text { - Convertirse en cuidador } \\
\text { - Existencia de co-morbilidad } \\
\text { como depresión o trastornos de } \\
\text { ansiedad } \\
\text { - Enfermedad orgánicas / médicas }\end{array}$ & $\begin{array}{l}\text { - Factores genéticos } \\
\text { - Resiliencia } \\
\text { - Fuerza de carácter } \\
\text { - Creencias religiosas } \\
\text { - Convicciones filosóficas }\end{array}$ & $\begin{array}{l}\text { - Vínculos de la infancia y } \\
\text { desarrollo familiar de la } \\
\text { autoestima } \\
\text { - Sucesos vitales / pérdidas } \\
\text { - Enfermedad médica }\end{array}$ \\
\hline
\end{tabular}

da debido al deterioro que conlleva la enfermedad; también se le puede restar importancia señalando que dada la situación de los pacientes, es comprensible y racional que presenten estos sentimientos de desesperanza y estrés emocional. Kissane, Clarke y Street ${ }^{(40)}$ además de proponer la existencia del síndrome, plantean la necesidad de diferenciarlo de otros fenómenos como son la abulia (asociada en depresión a una pérdida neurológica de motivación), la apatía (en el síndrome de desmoralización, la baja motivación es importante pero no es rasgo principal como sí ocurre en la depresión), el aburrimiento (definido como un sentimiento derivado de la monotonía sensorial y reducción de la vida interna e imaginación, algo frecuente ante el deterioro clínico en pacientes paliativos) y la vergüenza (relacionada por la pérdida de la autonomía, dependencia y necesidad de ayuda para las actividades de la vida diaria).

Es interesante notar como a pesar de la existencia de esta línea de trabajo sobre el síndrome de desmoralización, en los últimos años el estudio del estado de ánimo de los pacientes paliativos se ha centrado en las necesidades espirituales, aunque encontramos estudios que aportan evidencia de la existencia de este trastorno, además de su mejor adecuación frente al diagnóstico de depresión. Robinson, Kissane, Brooker y Burney ${ }^{(58)}$ han realizado una revisión recientemente sobre la investigación del síndrome de desmoralización. Siguiendo la metodología PRISMA, analizaron los resultados de 25 estudios (33 artículos). A partir de esta revisión, se encuentra que la prevalencia de la desmoralización evaluada con el DCPR se encontraba en un rango de $20,6 \%$ a $33,33 \%$. En los estudios en los que se había empleado la ED, el rango encontrado fue de $13 \%$ a $18 \%$. Además, se describieron las relaciones entre desmoralización factores sociodemográficos: estado civil (menos desmoralización en pacientes con pareja), situación laboral (mayor desmoralización en pacientes desempleados), edad (no se encontró ningún 
tipo de relación), sexo (mayor presencia de desmoralización en mujeres que en hombres, aunque dos estudios no informaron de diferencias) y nivel cultural (mayor desmoralización en pacientes con nivel

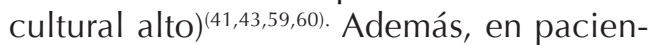
tes oncológicos avanzados se encontró una relación entre desmoralización y la presencia de síntomas físicos, aunque no en función del tipo de tumor ${ }^{(41,60,61)}$. También se encontraron relaciones entre desmoralización y depresión ${ }^{(44,53,56,62)}$. En cuanto a las relaciones entre desmoralización y otros factores psicológicos, se encontraron resultados que mostraban una relación inversa entre desmoralización y calidad de vida, esperanza y sentido de la $\operatorname{vida}^{(43,44,53,56,59,62) \text {. }}$.

Diagnóstico diferencial entre depresión y síndrome de desmoralización

A pesar de encontrarse rasgos distintos en estos trastornos, el diagnóstico diferencial entre ambos no parece ser fácil. Diferentes acercamientos se han llevado a cabo en estos años. Snaith ${ }^{(63)}$ señala como características básicas de la depresión la anhedonia, la pérdida de placer o interés en las actividades de la vida presente y futuras. Por otro lado Klein, Gittelman, Quitkin y Rifkin ${ }^{(64)}$ señalan que la característica básica de la desmoralización es la pérdida de sentido y la desesperanza, en la que la persona puede disfrutar de las actividades que realiza pero pierde el placer anticipatorio. Por otra parte, Parker ${ }^{(65)}$ distingue entre melancolía o depresión endógena (cambios motores en expresiones, gestos, discurso, etc.) y desmoralización (interés en lo cognitivo y afectivo pero sin los aspectos motores de la melancolía). En este sentido, Clarke, Kissane, Trauer y Smith ${ }^{(66)}$ Ilevaron a cabo un estudio con 271 pacientes con enfermedades físicas avanzadas (134 con trastorno motoneuronal y 137 con cáncer avanzado) en el que se administró una entrevista psiquiátrica estructurada. Los resultados que se encontraron en este estudio fueron que el $13,2 \%$ de la varianza se explicaba por el factor de desmoralización; el $8,3 \%$ de la varianza por la depresión anhedónica; el 6,8\% de la varianza por síntomas somáticos y el 53\% de la varianza, por el malestar/duelo. Tras llevar a cabo un análisis de regresión, se encontró además que la desmoralización estaba significativamente más presente en pacientes con trastorno motoneuronal y la anhedonia más presente en oncológicos.

En general, se aprecia que la persona con desmoralización puede sonreír, reír, expresar un rango amplio de afectos apropiados al contexto; pueden además informar de actividades con las que disfrutan y por las que tienen un interés normal, lo que marca una diferencia importante con el criterio del DSM-V de depresión mayor. Puede existir una comorbilidad entre depresión y desmoralización y a la vez, independencia entre ambas.

Kissane et al. ${ }^{(38)}$ proponen las siguientes nueve criterios para el diagnóstico de la desmoralización:

1. Pérdida de sentido de la vida

2. Desesperanza, fracaso personal, o carecer de un futuro que vale la pena

3. Sensación de estar atrapado o pesimismo

4. Sensación de renuncia

5. Desmotivación o incapacidad de afrontamiento

6. Aislamiento o alienación

7. Potencial pensamiento suicida

8. Fluctuación de la intensidad emocional,

9. Estos fenómenos persisten a lo largo de más de dos semanas

Así pues, parecen tener sentido los diagnósticos diferenciales del Síndrome de Desmoralización con Trastorno adap- 
tativo (con curso depresivo), Episodio Depresivo Mayor, Trastorno distímico, Trastorno del estado de ánimo inducido por sustancias, Trastorno afectivo por causa médica y Decatexis.

Síndrome de Desmoralización, necesidades espirituales y dignidad

La última década ha visto una gran incremento de la investigación en relación con las dimensiones espirituales del sufrimiento, así como nuevos enfoques para evaluar y mitigar este tipo de sufrimiento en pacientes de cuidados paliativos ${ }^{(67,68)}$. El número de trabajos que se pueden encontrar sobre este contenido a partir de 2005 ha ido creciendo de manera exponencial siendo hoy por hoy una dimensión de atención fundamental y prioritaria en estudio e intervención. Padecer una enfermedad grave, avanzada y en su fase terminal, puede hacer emerger necesidades espirituales hasta entonces latentes, que si no son satisfechas adecuadamente darán lugar al "sufrimiento espiritual" descrito por Saunders ${ }^{(68)}$. Pacientes con las necesidades espirituales cubiertas presentan niveles bajos de ansiedad y depresión ${ }^{(41,70)}$. En sentido contrario, Mehnert et al ${ }^{(71)}$ y Passik ${ }^{(72)}$ encontraron que pacientes con problemas espirituales presentaban niveles más altos de desmoralización. Por otro lado, Clarke y Boscaglia ${ }^{(50)}$ no encontraron una relación directa entre desmoralización y espiritualidad pero sí una relación importante entre desmoralización y pérdida de sentido. Podríamos afirmar que el trabajo sobre las necesidades espirituales ha sustituido en cierta medida al de la desmoralización del paciente paliativo.

En su trabajo en relación con el deseo de vivir entre los pacientes con cáncer avanzado, Chochinov et al. ${ }^{(73)}$ destacaron el papel fundamental de cuestiones tales como la desesperanza, la percepción de uno mismo como una carga para los de- más y la vulneración de la dignidad como factores cardinales que influyen en la pérdida del deseo de vivir. La construcción de la esperanza conecta con un sentido de significado y propósito; en la literatura científica se evidencia que perder el sentido de significado y propósito y experimentar la vida como redundante o inútil son importantes fuentes de angustia que contribuyen a la pérdida de las ganas de vivir. La atención a la percepción de una muerte digna por parte del paciente paliativo se refleja en el volumen de trabajos que se encuentran publicados en los últimos años ${ }^{(3,7,42,74-79)}$. Posiblemente el modelo planteado por Chochinov et al..$^{(74)}$ es el que cuenta con un mayor desarrollo. Podría definirse como un marco terapéutico adecuado para atender al paciente moribundo y su familia, de carácter obligatorio y no discutible. Este marco terapéutico se articula en tres categorías ${ }^{(74)}$ : Preocupaciones relacionadas con la enfermedad, Preservación de la dignidad (personal) y Preservación de la dignidad (social).

Las relaciones y semejanzas entre desmoralización, necesidades espirituales y dignidad son evidentes. En la Tabla 6 pueden encontrarse los criterios y dimensiones de cada uno de los constructos.

El conjunto de estas semejanzas nos está señalando a los clínicos que nuestro abordaje es mucho más complejo y de alcances mucho más amplios de lo que inicialmente podría parecer. Tratar el estado de ánimo de una manera adecuada puede suponer a la par la atención de las necesidades espirituales del paciente y esto puede revertir en la percepción de la preservación de la dignidad al final de la vida. Estas relaciones no invalidan ningún abordaje, más bien refuerzan la necesidad de diagnosticar al paciente paliativo en cuanto a su malestar emocional y el síndrome de desmoralización parece ser un buen punto de partida para, una vez identificado, facilitar su manejo. 


\section{Tabla 6. Relaciones y diferencias entre Desmoralización, Espiritualidad y Dignidad}

\begin{tabular}{|c|c|c|}
\hline Desmoralización ${ }^{38}$ & $\begin{array}{l}\text { Espiritualidad (Dimensiones } \\
\text { Grupo SECPAL) }{ }^{87}\end{array}$ & Dignidad 74 \\
\hline $\begin{array}{l}\text { Pérdida de sentido de la } \\
\text { vida } \\
\text { Desesperanza, fracaso } \\
\text { personal, o que carecen de } \\
\text { un futuro que vale la pena } \\
\text { Sensación de estar atrapado } \\
\text { o pesimismo } \\
\text { Sensación de renuncia } \\
\text { Desmotivación o } \\
\text { incapacidad de } \\
\text { afrontamiento } \\
\text { Aislamiento o alienación } \\
\text { Potencial pensamiento } \\
\text { suicida } \\
\text { Fluctuación de la } \\
\text { intensidad emocional, } \\
\text { Estos fenómenos persisten a } \\
\text { través de más de } 2 \text { semanas }\end{array}$ & $\begin{array}{l}\text { Dimensión intrapersonal } \\
\text { (relaciones con uno mismo, } \\
\text { satisfacción, sensación de } \\
\text { logro, necesidades: pérdida } \\
\text { de autonomía, dependencia, } \\
\text { fracaso). } \\
\text { - Dimensión interpersonal } \\
\text { (nuestras relaciones con } \\
\text { otros, sentirse querido, } \\
\text { sentirse en paz con los } \\
\text { demás, necesidades: } \\
\text { aislamiento social). } \\
\text { - Dimensión transpersonal } \\
\text { (conciencia de pertenencia a } \\
\text { una dimensión trascendente, } \\
\text { confianza, esperanza, } \\
\text { legado, conexión con } \\
\text { una realidad superior, } \\
\text { necesidades: desesperanza, } \\
\text { pérdida sentido vida, } \\
\text { impotencia). }\end{array}$ & $\begin{array}{l}\text { Preocupaciones relacionadas } \\
\text { con la enfermedad (nivel } \\
\text { de independencia: agudeza } \\
\text { cognitiva y capacidad } \\
\text { funcional; síntomas de } \\
\text { malestar: malestar físico y } \\
\text { malestar psicológico) } \\
\text { - Preservación de la dignidad } \\
\text { (personal). Perspectivas } \\
\text { que conservan la dignidad } \\
\text { (continuidad del yo, conservar } \\
\text { los roles, dejar un legado, } \\
\text { mantener el orgullo, la } \\
\text { esperanza, autonomía / } \\
\text { control, aceptación, } \\
\text { resistencia / espíritu de lucha) } \\
\text { Preservación de la dignidad } \\
\text { (social). Esfera de privacidad } \\
\text { (apoyo social, calidad del } \\
\text { cuidado, carga de los otros, } \\
\text { preocupaciones con el legado) }\end{array}$ \\
\hline
\end{tabular}

\section{CONCLUSIONES}

Es innegable que existen ciertas dificultades para el diagnóstico de los trastornos del estado de ánimo en pacientes paliativos y esta realidad afecta a los tratamientos y a los resultados esperados de estos. Aunque el diagnóstico de Síndrome de Desmoralización aún no se encuentra reconocido en el DSM-V, existen datos convincentes para apoyar la existencia de un "síndrome de desmoralización" que es clínicamente distinguible de la depresión y la ansiedad. Este síndrome puede ser una fuente común de estrés psicológico y existencial en pacientes con enfermedades graves y avanzadas como el cáncer ${ }^{(45)}$. La desmoralización se define como la conciencia de incapa- cidad para hacer frente a un problema grave y se caracteriza por sentimientos de impotencia, desesperanza, incompetencia subjetiva y pérdida de control.

Los datos reflejados en la investigación sugieren que este tipo de angustia se asocia comúnmente con enfermedad crónica física y terminal, discapacidad, desfiguración corporal, miedo a la pérdida de la dignidad, el aislamiento social y la sensación de ser una carga para los demás, todos ellos aspectos presentes en los cuidados paliativos.

La característica fundamental para distinguir la desmoralización de la depresión es la ausencia de anhedonia: los pacientes con síndrome de desmoralización, aunque profundamente pesimistas hacia el futuro, pueden ser capaces de obtener 
placer de situaciones reales o imaginarias y de las interacciones satisfactorias.

La principal diferencia entre la depresión y el síndrome de desmoralización se encuentra en los síntomas somáticos. Para el diagnóstico de la depresión, estos síntomas cuentan con un peso igual de importante que los cognitivos, no sucediendo así en el caso de la desmoralización. El planteamiento de la desmoralización en pacientes paliativos hace hincapié en los aspectos emocionales y cognitivo experienciales dado que los síntomas físicos son de naturaleza patológica, es decir, con una base fundamentalmente orgánica y no cognitiva o afectiva.

Se entiende que el síndrome de desmoralización es un buen reflejo del estado de ánimo del paciente paliativo, donde la desesperanza, o los sentimientos de dependencia, aspectos estos más vivenciales, más cognitivos y emocionales, se perfilan como determinantes más pertinentes que la pérdida de peso o el insomnio, tanto en la descripción de su estado de ánimo como en el diagnóstico subsiguiente.

Es necesario señalar que aun asumiendo que el estado de ánimo deprimido puede ser una respuesta adaptativa o normalizada en el paciente paliativo, no se debe considerar como «normal» cualquier manifestación de tristeza en sus diferentes grados. Los clínicos han de tener en cuenta el contexto en que se dan los síntomas, su adecuación, así como la gravedad de los mismos. Si fuera de otro modo, dejaríamos sin apoyo a muchas personas que sufren. Algunos autores cuestionan la existencia de dicho síndrome, pero consideramos que las evidencias prácticas del trabajo con distintas intervenciones psicológicas como son el counselling o la terapia de la dignidad $^{(3,4,6,42)}$ obligan al estudio de este cuadro, ya que la no detección adecuada de necesidades emocionales del paciente puede llevar a que éste no reciba un tratamiento psicológico necesario.
A pesar del trabajo desarrollado respecto a la desmoralización, existen diferencias en cuanto a criterios del propio trastorno que deben resolverse y ajustarse, para validar el cuadro sintomático y poder ser, si procede, reconocido dentro de los manuales diagnósticos como el DSM.

Una conclusión importante que se desprende de la existencia del síndrome de desmoralización es el planteamiento de una serie de objetivos terapéuticos concretos y diferentes a los habituales. Este hecho nos lleva de inmediato a desarrollar terapéuticas diferentes y por lo tanto, a esperar unos resultados que van encaminados precisamente a esos objetivos, alineados con el cuadro de desmoralización. Es necesario pues, continuar con el esfuerzo realizado por encontrar los criterios diagnósticos adecuados y planteados como categoría diagnóstica dentro del marco del síndrome de desmoralización, que parece englobar las características específicas de los pacientes en la fase final de la vida. Todo ello tiene repercusiones importantes no sólo a nivel teórico, sino también en los resultados que puedan encontrarse en el estudio de la dignidad y de las dimensiones espirituales de los pacientes paliativos arrojando luz sobre las coincidencias con el concepto de desmoralización.

En todo plan de intervención, la fase de evaluación es fundamental, pues permite establecer el plan terapéutico. Lamentablemente, el cuadro sintomático del estado de ánimo del paciente paliativo parece estar abordándose desde una perspectiva diferente, lo que puede suponer no solo que no se esté tratando adecuadamente al paciente, sino que se pueden estar obviando algunas intervenciones que pueden proporcionar calidad de vida al paciente. Es necesario seguir trabajando en la línea de evaluar lo que realmente siente el paciente paliativo y aportar evidencias de que los tratamientos se adecuan a sus necesidades. 


\section{REFERENCIAS BIBLIOGRÁFICAS}

1. Kübler-Ross E. Sobre la muerte y los moribundos. Barcelona: Editorial Grijalbo; 1989.

2. Romero C, Álvarez M, Bayés R, Schröder M. ¿Cuándo se pide la intervención del psicólogo en una unidad de cuidados paliativos? Med Paliat 2001;8:170-2.

3. Julião M, Oliveira F, Barbosa A. Efficacy of dignity therapy in the anxiety of terminaIly III patients: randomized controlled trial. Palliat Support Care 2013;11:481-9. Doi: 10.1017/S147895151200089

4. Julião M, Oliveira F, Nunes B, Vaz Carneiro A, Barbosa A. Efficacy of dignity therapy on depression and anxiety in Portuguese terminally ill patients: A phase II randomized controlled trial. J PaIliat Care 2014;17:688-95. Doi:10.1089/ jpm.2013.0567.

5. Houmann LJ, Cochinov HM, Kristjanson LJ, Petersen MA, Groenvold M. A prospective evaluation of Dignity Therapy in advanced cancer patients admitted to paIliative care. Palliat Med 2014;28:448-58. Doi:10.1177/0269216313514883

6. Rudilla, D, Barreto P, Oliver A, Galiana L. Eficacia del counselling y la terapia de la dignidad en pacientes paliativos. Med Paliativa 2015;22. Doi: 10.1016/j.medipa.2014.09.005. En prensa.

7. Rudilla, D, Barreto P, Oliver A, Galiana L, Ruiz A. Eficacia de la terapia de la dignidad en pacientes paliativos en atención domiciliaria". X Congreso Nacional de la Sociedad Española de Cuidados Paliativos. 2014 Nov 13-15; Madrid

8. Rudilla, D, Barreto P, Oliver A, Galiana L, Ruiz A. Eficacia del counselling en pacientes paliativos en atención domiciliaria. X Congreso Nacional de la Sociedad Española de Cuidados Paliativos; 2014 Nov 1315; Madrid.

9. Tremblay A'Breitbart W. Psychiatric dimensions of palliative care. Neurol Clin 2001;19:949-67.
10. Lichtenthal WG, Nilsson M, Zhang B, Trice ED, Kissane DW, Breitbart W, et al. Do rates of mental disorders and existential distress among advanced stage cancer patients increase as death approaches? Psychooncology 2009;18:50-61. Doi: 10.1002/pon.1371.

11. Brugha T. Depression in the terminally ill. Br J Hosp Med 1993;50:177-181.

12. Chochinov HM, Wilson KG, Enns M, Lander S. Are you depressed? Screening for depression in the terminally ill. Am J Psychiatry 1997; 154:674-6.

13. Lloyd-Williams M, Payne S. Nurse specialist assessment and management of palliative care patients who are depressed- a study of perceptions and attitudes. J Palliat Care 2002;18:270-4.

14. Passik S.D, Dugan W, McDonald MV, Rosenfeld B, Theobald D, Edgerton, S. Oncologists' recognition of depression in their patients with cancer. J Clin Oncol 1998;16:1594-600.

15. Mitchell AJ, Chan $M$, Bhatti $H$, Halton $M$, Grassi L, Johansen C, Meader N. Prevalence of depression, anxiety, and adjustment disorder in oncological, haematological, and palliative-care settings: A meta-analysis of 94 interview-based studies. Lancet Oncol 2011;12:160-74. Doi: 10.1016/ S1470-2045(11)70002-X.

16. Rajmohan, V., y Kumar, K. S. (2013). Psychiatric morbidity, pain perception, and functional status of chronic pain patients in palliative care. Indian J Palliat Care 2013;19:14651. Doi: 10.4103/0973-1075.121527.

17. Breitbart W, Jaramillo JR, Chochinov HM. Palliative and terminal care. En: Holland JC, editor. Psycho-oncology. New York: Oxford University Press; 1998. p.437-49.

18. Wilson KG, Cochinov HM, de Faye BJ, Breitbart W. Diagnosis and Management of Depression in Palliative Care. En: Chochinov HM, Breitbart W, editors. Handbook of Psychiatry in Palliative Medicine. New York: Oxford University Press; 2000. p.25-49. 
19. Cox J, Holden J, Sagovsky R. Detection of postnatal depression: development of 10 item Edinburgh Postnatal Depression Scale. Br J Psychiatry 1987;150:782-6.

20. Lloyd-Williams M, Friedman T, Rudd N. Criterion validation of the Edinburgh Postnatal Depression Scale as a screening tool for depression in patients with advanced metastatic cancer. J Pain Symptom Manage 2000;20:259-65.

21. Tejero A, Guimera E, Farré JM, Peri JM. Uso clínico del HADS (Hospital Anxiety and Depression Scale) en población psiquiátrica: un estudio de su sensibilidad, fiabilidad y validez. Revista Departamento Psiquiatría 1986;13,233-338.

22. Thekkumpurath $\mathrm{P}$, Venkateswaran $\mathrm{C}, \mathrm{Ku}$ mar M, Bennett MI. Screening for psychological distress in palliative care: Performance of touch screen questionnaires compared with semistructured psychiatric interview. J Pain Symptom Manage 2009;38:597-605. Doi: 10.1016/j.jpainsymman.2009.01.004.

23. Julião $M$, Barbosa $A$. Depressão em cuidados paliativos. Acta Med Port 2011; 24(S4):807-18.

24. Hotopf M, Chidgey J, Addington-Hall J, Lan Ly K. Depression in advanced diseased: A systematic review. Part 1. Prevalence and case finding. Palliat Med 2002;16:81-97.

25. Lloyd-Williams M, Friedman T, Rudd N. An analysis of the Validity of the Hospital Anxiety and Depression Scale as a screening tool in patients with advanced metastatic cancer. J Pain Symptom Manage 2001;22:990-6.

26. Le Fevre P, Devereux J, Smith S, Lawrie SM, Cornbleet M. Screening for psychiatric illness in palliative care inpatient setting: A comparison between the Hospital Anxiety and Depression Scale and the General Health Questionnaire- 12. Palliat Med 1999;13:399-407.

27. Holtom N, Barraclough J. Is the Hospital Anxiety and Depression Scale (HADS) useful in assessing depression in palliative care? Palliat Med 2000;14:219-20.
28. Hamilton M. Development of a rating scale for primary depressive illness. Br 」 Soc Clin Psychol 1967;6:278-96.

29. Chochinov HM, Wilson KG, Enns M, Lander S. Prevalence of depression in the terminally ill: Effects of diagnostic criteria and symptom threshold judgments. Am J Psychiatry 1994;151:537-40.

30. Muñiz J, Fernández-Hermida JR. La opinión de los psicólogos españoles sobre el uso de los test. Papeles del Psicólogo 2010;31:108-21.

31. Mahoney J, Drinka TJ, Abler R, GunterHunt G, Matthews C, Gravenstein S, Carnes M. Screening for depression: Single question versus GDS. J Am Geriatr Soc 1994;42:1006-8.

32. Coates A, Dillenbeck CF, McNeil DR, Kaye SB, Sims K, Fox RM, et al. On tje receiving end: Linear analogue self-assessment (LASA) in evaluaton of aspects of the quality of life of cancer patients receiving therapy. Eur J Cancer Clin Oncol 1983;19:1633-7.

33. Lees N, Lloyd-Williams M. Assessing depression in palliative care patients using the Visual Analogue Scale: A pilot study. Eur J Cancer Care (Engl) 1999;8:220-3.

34. Chochinov HM, Kristjanson L, Breitbart W, McClement S, Hack T, Hassard T, Harlos $M$. The effect of dignity therapy on distress and end-of-life experience in terminally ill patients: a randomised controlled trial. Lancet Oncol 2011;12:753-62. Doi: 10.1016/S1470-2045(11)70153-X

35. Derogatis LR, Melisaratos N. The Brief Symptom Inventory: An introductory report. Psychol Med 1983;13:595-605.

36. Ruipérez, MA, Ibáñez MI, Lorente E, Moro M, Ortet G. Psychometric properties of the Spanish version of the BSI: Contributions to the relationship between personality and psychopathology. Eur J Psychol Assess 2001; 17:241-50

37. Jacobsen PB, Donovan, KA, Trask PC, Fleishman SB, Zabora, J, Baker F, Holland JC. Screening for psychologic dis- 
tress in ambulatory cancer patients. Cancer 2005;103:1494-502. Doi: 10.1002/ cncr.20940

38. Kissane DW, Clarke DM, Street AF. Demoralization syndrome-a relevant psychiatric diagnosis for palliative care. J Palliat Care 2001;17:12-21.

39. Portela MA, Rubiales AS, Centeno C.. The use of psychostimulants in cancer patients Curr Opin Support Palliat Care 2011;5:164-

8. Doi: 10.1097/SPC.0b013e3283462ff3.

40. Priede A, Ruiz M, Hoyuela F, Herrán A, González-Blanch C. El termómetro del distréss como prueba de cribado de ansiedad y depresión en pacientes oncológicos recién diagnósticos. Psicooncología 2014;11:31-43. Doi: 10.5209/ rev_PSIC.2014.v11.n1.44915

41. Morita $T$, Kawa M, Honke $Y$, Kohara $H$, Maeyama E, Kizawa Y, et al. Existential concerns of terminally ill cancer patients receiving specialized palliative care in Japan. Support Care Cancer 2004;12:13740. Doi: 10.1007/s00520-003-0561-6

42. Mehnert A, Vehling S, Hocker A, Lehmann $C$, Koch U. Demoralization and depression in patients with advanced cancer: Validation of the German version of the demoralization scale. J Pain Symptom Manage 2011;42:768-76. Doi: 10.1016/j. jpainsymman.2011.02.013

43. Julião $M$, Barbosa $A$, Oliveira $F$, Nunes B, Carneiro A. Efficacy of dignity therapy for depression and anxiety in terminally ill patients: Early results of a randomized controlled trial. Palliat Support Care. 2013;11:481-9. Doi: 10.1017/ S1478951512000892

44. Lee CY, Fang CK, Yang YC, Liu CL, Leu YS, Wang TE, et al. Demoralization syndrome among cancer outpatients in Taiwan. Support Care Cancer 2012; 20:2259-67. Doi: 10.1007/s00520-011-1332-4.

45. Vehling S, Lehmann C, Oechsle K, Bokemeyer C, Krüll A, Koch U, et al. Is advanced cancer associated with demoralization and lower global meaning? The role of tumor stage and physical problems in explaining existential distress in cancer patients. Psychooncology 2012,21:54-63. Doi: 10.1002/pon.1866.

46. Fang CK, Chang MC, Chen PJ, Lin CC, Chen GS, Lin J et al. A correlational study of suicidal ideation with psychologicaL distress, depression, and demoralization in patients with cancer. Support Care Cancer 2014;22:3165-74. Doi: 10.1007/s00520-014-2290-4.

47. Clarke DM, Kissane DW. Demoralization: its phenomenology and importance. Aust N Z J Psychiatry 2002;36:733-42.

48. Clarke DM, Mackinnon AJ, Smith GC, McKenzie DP, Hermna HE. (2000). Dimensions of psychopatology in the medically ill: A latent trait analysis. Psychosomatics 2000;41:418-25.

49. Frank JD. Psychotherapy: The restoration of morale. Am J Psychiatr 1974;131:271-4.

50. de Figueiredo JM. Depression and demoralization: Phenomenological differences and research perspectives. Compr Psychiatr 1993;34:308-11. Doi: 10.1016/0010-440x(93)90016-w

51. Zautra AJ, Guarnaccia CA, Reich JW. Factor structure of mental health measures for older adults. J Consul Clin Psych 1988;56:514-519.

52. Dohrenwend BP, Shrout PE, Ergi G, Mendelsonh FS. Nonspecific psychological distress and other dimensions of psychopathology. Arch Gen Psychiatry 1980;37:1229-36.

53. Kissane DW, Wein S, Love A, Lee XQ, Kee $\mathrm{PL}$, Clarke DM. The Demoralization Scale: A report of its development and preliminary validation. J Palliat Care 2004;20:269-76.

54. Fava GA, Freyberger HJ, Bech P, Christodoulou G, Sensky T, Theorell T et al. Diagnostic criteria for use in psychosomatic research. Psychother Psychosom 1995;63:1-8.

55. Cockram CA, Doros G, De Figueiredo JM. Diagnosis and measurement of subjective incompetence: The clini- 
cal hallmark of demoralization. Psychother Psychosom 2009;78:342-5. Doi: $10.1159 / 000235737$.

56. Constantini A, Picardi A, Brunnetti S, Trabuchhi G, Bersani FS, Minichino A, Marchetti O. Italian version of Demoralization Scale: A validation study. Riv Psichiatr 2013;48:234-9. Doi: 10.1708/1292.14291.

57. Kissane DW, Treece C, Breitbart W, McKeen NA, Chochinov HM. Dignity, meaning and demoralization: Emerging paradigms in end-of-life-care. In: Chochinov HM, Breitbart W, editors. Handbook of psychiatry in palliative medicine. New York: Oxford University Press; 2009. p. 324-340.

58. Robinson S, Kissane DW., Brooker J, Burney S. A review of the construct of demoralization: History, definitions, and future directions for palliative care. Am J Hosp Palliat Care 2014 Oct 7. p: 1049909114553461. Doi: 10.1177/1049909114553461

59. Grandi S, Sirri L, Tossani E, Fava GA. Psychological characterization of demoralization in the setting of heart transplantation. J Clin Psychiatry 2011;72:648-54. Doi: 10.4088/JCP.09m05191blu

60. Katz RC, Flasher L, Cacciapaglia H, Nelson S. The psychosocial impact of cancer and lupus: a cross validation study that extends the generality of "benefit finding" in patients with chronic disease. J Behav Med 2001;24:561-71.

61. Boscaglia N, Clarke DM. Sense of coherence as a protective factor for demoralisation in women with a recent diagnosis of gynaecological cancer. Psychooncology 2007;16:189-95.

62. Grassi L, Rossi E, Sabato S, Cruciani G, Zambelli M. Diagnostic criteria for psychosomatic research and psychosocial variables in breast cancer patients. Psychosomatics 2004;45:483-91

63. Snaith P. Anhedonia: A neglected symptom of psychopathology. Psychol Med 1993;23:957-66.
64. Gittelman R. Diagnosis and Drug Treatment of Childhood Disorders. In: Klein DF, Gittelman, R, Quitkin, F, Rifkin, A, editors. Diagnosis and drug treatment of psychiatric disorders: adults and children. Baltimore: Williams \& Wilkins, 1980. p. 590-775.

65. Parker M. Medicalizing meaning: Demoralization syndrome and the desire to die. Aust N Z J Psychiatry 2004;38:765-73.

66. Clarke DM, Kissane DW, Trauer T, Smith GC. Demoralization, anhedonia and grief in patients with severe physical illness. World Psychiatry 2005;4:96-105.

67. Whitford HS, Olver IN, Peterson MJ. Spirituality as a core domain in the assessment of quality of life in oncology. Psychooncology 2008;17:1121-8. Doi: 10.1002/ pon.1322.

68. Vachon M, Fillion L, Achille M. A conceptual analysis of spirituality at the end of life. J Palliat Med 2009;12:53-9. Doi: 10.1089/jpm.2008.0189.

69. Saunders C. Spiritual Pain. J Palliat Care 1988;4:29-32.

70. Barreto P, Fombuena M, Diego R, Galiana L, Oliver A, Benito E. Bienestar emocional y espiritualidad al final de la vida. Med Paliat 2015;22:25-32.

71. Rudilla D. Elaboración y estudio de una herramienta de evaluación de la dignidad al final de la vida [tesis]. Valencia: Universitat de València; 2014.

72. Passik SD, Inman A, Kirsh K, Theobald D, Dickerson P. Initial validation of a scale to measure purposelessness, understimulation, and boredom in cancer patients: Toward a redefinition of depression in advanced disease. Palliat Support Care 2003;1:41-50.

73. Chochinov HM, Hack T, Hassard T, Kristjanson LJ, McClement S, Harlos M. Understanding the will to live in patients nearing death. Psychosomatics 2005;46:7-10.

74. Chochinov HM, Hack T, McClement S, Kristjanson L, Harlos M. Dignity in the terminally ill: a developing empirical model. Soc Sci Med 2002;54:433-43. 
75. Jors K, Adami S, Xander C, Meffert C, Gaertner J, Bardenheuer $\mathrm{H}$, et al. Dying in cancer centers: Do the circumstances allow for a dignified death? Cancer 2014;120:3254-60. Doi: 10.1002/ cncr.28702.

76. Guo C, Jacelon C. An integrative review of dignity in end-of-life care. Palliat Med 2014;31;28:931-40.

77. Jacelon CS, Choi J. Evaluating the psychometric properties of the Jacelon Attributed Dignity Scale. J Adv Nurs 2014;70:214961. Doi: 10.1111/jan.12372.

78. Oosterveld-Vlug MG, Onwuteaka-Philipsen BD, Pasman HR, van Gennip IE, de Vet HC. Can personal dignity be assessed by others? A survey study comparing nursing home residents' with family members', nurses' and physicians' answers on the MIDAM-LTC. Int J Nurs Stud 2015;52:555-67. Doi: 10.1016/j.ijnurstu.2014.06.007.

79. Lindqvist O, Threlkeld G, Street AF, Tishelman C. Reflections on using biographical approaches in end-of-life care: Dignity Therapy as example. Qual Health Res 2015;25:40-50. Doi: 10.1177/1049732314549476.

80. American Psychiatric Association. DSM-5. Manual diagnóstico y estadístico de los trastornos mentales. Editorial: Editorial Médica Panamericana; 2014.

81. D'Arcy C. Prevalence and correlates of nonpsychotic psychiatric symptoms in the general population. Can J Psychiatry 1982;27:316-24.

82. Clarke DM, Smith GC, Dowe DL, McKenzie DP. An empirically derived taxonomy of common distress syndromes in the medicaIly ill. J Psychosom Res 2003;54:323-30.

83. Griffith JL, Gaby L. Brief psychotherapy at the bedside: Countering demoralization from medical illness. Psychosomatics 2005:46:109-16.Doi: 10.1176/appi. psy.46.2.109

84. Clarke DM, Cook KE, Coleman KJ, Smith GC. A qualitative examination of the experience of "depression" in hospitalized medical ill patients. Psychopathology 2006;39:303-12.

85. Hung HC, Chen HW, Chang YF, Yang $\mathrm{YC}$, Liu CL, Hsieh RK, et al. Evaluation of the reliability and validity of the Mandarin Version of Demoralization Scale for cancer patients. J Intern Med Taiwan 2010;21:427-35

86. Mullane M, Dooley B, Tiernan E, Bates $U$. Validation of the Demoralization Scale in an Irish advanced cancer sample. PaIliat Support Care 2009;7:323-30. Doi: 10.1017/S1478951509990253.

87. Benito E, Oliver A, Galiana L, Barreto P, Pascual A, Gomis C, et al. Development and validation of a new tool for the assessment and spiritual care of palliative care patients. J Pain Symptom Manage 2014;47:1008-18. Doi: 10.1016/j. jpainsymman.2013.06.018. 